\title{
AVALIAÇÃO DO GRAU DE EXPOSIÇÃO DE AMOSTRAS POPULACIONAIS DE SÃO PAULO (BRASIL) AO MONÓXIDO DE CARBONO *
}

Nilda A. Gallego Gandara de Fernicola**

Ernesto Ronchini Lima**

Fernicola, N. A. G. G. de \& Lima, E. R. Avaliação do grau de exposição de amostras populacionais de São Paulo ao monóxido de carbono. Rev. Saúde públ., S. Paulo, 13:151-8, 1979.

Resumo: Com a finalidade de avaliar o grau de exposição ao monóxido de carbono da população da cidade de São Paulo (Brasil), foram coletadas 327 amostras de sangue de adultos, procedendo-se à determinação do conteúdo de carboxiemoglobina pela técnica espectrofotométrica. Trinta das amostras obtidas de residentes de uma cidade (Embú-Guaçu) considerada, por suas caracteristicas, como local de baixas concentrafóes de monóxido de carbono, foram tomadas como grupo-controle. Os valores de carboxiemoglobina encontrados foram os seguintes: policiais de trânsito: fumantes $6,3 \pm 2,07$, não fumantes $2,1 \pm 0,68$; motoristas de ônibus: fumantes $4,6 \pm 1,94$, não fumantes $1,6 \pm 0,46 ;$ grupocontrole: fumantes $3,8 \pm 1,74$, não fumantes $0,8 \pm 0,21$.

UNITERMOS: Monóxido de carbono. Espectrofotometria.

\section{N TRODUIC AO}

O monóxido de carbono ( $\mathrm{CO}$ ) é quase inteiramente produzido e emitido na atmosfera como sub-produto da combustão incompleta de combustiveis fósseis.

Os automotores são responsáveis pela emissão de cerca de $75 \%$ do CO produzido por fontes artificiais, mundialmente.

A toxicidade do $\mathrm{CO}$ é devida às suas reações de combinação reversivel com as hemoproteinas tais como a mioglobina, citocromo oxidase, citocromo $P_{450}$, hidroxiperoxidases. Entretanto, é com a hemoglobina que fica caracterizada sua combinação principal, pois a afinidade desta hemoproteína com o CO é cerca de 210 vezes maior que com o oxigênio.

Pode-se afirmar que as combinações de $\mathrm{CO}$ com as outras hemoproteinas já citadas, em conjunto, representam somente de 10 a $15 \%$ do total de $\mathrm{CO}$ no homem normal, atribuindo-se 0 resto à carboxiemoglobina $(\mathrm{COHb})^{1+}$.

A produção endógena de $\mathrm{CO}$ no corpo humano é devida ao catabolismo normal do heme, atingindo-se cerca de $0,4 \mathrm{ml}$ de $\mathrm{CO}$ por hora, os quais resultam, para adultos

* Trabalho realizado na Companhia de Tecnologia de Saneamento Ambiental (CETESB).

* Da Diretoria de Tecnologia de Saneamento do Ar e de Controle de Fontes de Poluição (DTSA) da CETESB - Av. Prof. Frederico Hermann Jr., 345 - 05459 - São Paulo SP - Brasil. 
FERNiCOLA, N. A. G. G. de \& LIMA, E. R. Avaliação do grau de exposição de amostras populacionais de São Paulo (Brasil) ao monóxido de carbono. Rev. Saúde públ., S. Paulo, 13: 151-8, 1979.

normais, em um percentual da saturação de $\mathrm{COHb}$ cle cerca de $0,5 \%{ }^{15}$.

Lafontaines destaca que a presença de $\mathrm{COHb}$ entre $0,5-0,8 \%$ poderia corresponder a uma exposição continua, a $5 \mathrm{ppm}$ de $\mathrm{CO}$ no ar, ou a uma exposição de 8 horas a 7 ppm ou uma hora a 20 ppm.

En termos de exposição ao $\mathrm{CO}$ exógeno, vários fatores influem sobre os percentuais de saturação de $\mathrm{COHb}$ atingidos, entre os quais destacamos: as pressões parciais do oxigênio e do $\mathrm{CO}$ no ambiente de exposição, o tipo de atividade física que se está desempenhando bem como o hábito de fumar. Estudos cuidadosos 10 têm demonstrado que a taxa de formação de $\mathrm{COHb}$ é diretamente proporcional à ventilação alveolar e ao gradiente das pressões parciais de $\mathrm{CO}$ estabelecido entre os alvéolos e os capilares pulmonares.

O homem é, em última instância, o indicador biológico da contaminação do ar, e atua como um mecanismo de amostragem. Assin, no referente a un dos contaminantes mais conhecidos e estudados, como é - CO, a determinação da quantidade deste no sangue de não fumantes, expressada como um volume do gás ou como percentagem de $\mathrm{COHb}$, permite conhecer o risco a que está sujeito o homem em relação a este contaminante ${ }^{11}$.

O CO é medido na cidade de São Paulo em um só lugar, no centro da cidade (Praça do Correio), sendo impossivel então relacionar as concentraçōes desse lugar com a ação deste contaminante sobre os habitantes. Entretanto, em se conhecendo os valores apresentados pelos grupos de exposição extremada poder-se-á inferir no limite superior de exposição da população em geral. Desta forma, procedeu-se ao presente trabalho, determinando a $\mathrm{COHb}$ expressa como percentual de saturação da hemoblobina nos grupos sabidamente mais expostos.

\section{MATERIAL E METODOS}

Sendo, como já referido, os veículos automotores os maiores responsáveis pelas emissões de CO, escolheram-se neste estudo grupos populacionais bem relacionados aos veículos e suas condições de tráfego, isto é, foram escolhidos os motoristas de ônibus, elétricos e a combustão, bem como policiais de trânsito na cidade de São Paulo, para constituirem o "grupo exposto". Tomou-se como grupo-controle policiais e funcionários públicos da cidade de Embu-Guaçú, onde não há razão para acreditar-se na existência de altas concentrações de $\mathrm{CO}$, seja emitido por veículos automotores ou por fontes estacionárias de combustão.

Foi analisado um total de 327 amostras, 297 das quais relativas ao "grupo exposto" e 30 ao grupo-controle. No momento da tomada das amostras foi preenchido um questionário onde constava: tipo de trabalho, horas trabalhadas neste dia, hábito de fumar, entre outros.

Geralmente a tomada das amostras for realizada ao redor do meio dia, o que representa de 6 a 7 horas de exposição.

As amostras de sangue foram obtidas por punção de dedo e o sangue recolhido em tubos capilares heparinizados, os quais foram mantidos em geladeira a $4^{\circ} \mathrm{C}$ e seu conteúdo analisado antes que se completassem dois dias de sua obtenção. Cada amostra foi colhida e analisada em triplicado.

A determinação da $\mathrm{COHb}$, expressada como percentual de saturação da hemoglobina, se realizou pela técnica de Buchwald ", a qual é uma simplificação da publicada por Commins e Lawther ${ }^{4}$.

Foi utilizado um espectrofotômetro Varian 635. Para leitura de absorbância foram usadas cubas de $1 \mathrm{~cm}$ de percurso ótico. O CO necessário para a análise das amostras foi obtido por reação entre o formiato de sódio e ácido sulfúrico concentrado e o oxigênio utilizado foi obtido de cilindro pressurizado. 
FERNiCola, N. A. G. G. de \& LiMA. E. R. A waliação do grau de exposição de amostras populacionais de São Paulo (Brasil) ao monóxido de carbono. Rev. Saúde públ., S. Paulo, 13: $151-8,1979$.

\section{RESULTADOS}

A população amostrada foi classificada segundo o hábito de fumar, devido a significância deste nos percentuais de $\mathrm{COHb}$ presentes no sangue, obtendu-se a distribuição que consta nas Tabelas 1 e 2 .

As amostras foram coletadas nos seguintes periodos:

São Paulo ("grupo exposto"):

cmpregados de transportes: de 10/11/76 a $20 / 12 / 76$

guardas de trânsito: de $26 / 01 / 77$ a $28 / 02 / 77$

Embu-Guaçú (grupo-controle) :

funcionários públicos e policiais: de 02/03/77 a 09/03/77.

Os valores de $\mathrm{COHb}$ obtidos constam nias Tabelas 3 e 4 .
Do estudo dos dados obtidos por inter. valo de classe foram construidos os histogramas das Figs. 1, 2, 3 e 4*.

Em termos de freqüência acumulada, a Fig. 5* mostra as distribuições percentuais obticlas para fumantes e não-fumantes do grupo controle bem como do "grupo exposto".

Pela análise da Fig. 5 verificon-se yue como o esperado, as curvas $a, c e d$ referuntes aos grupos não-fumantes estão deslocadas para a esquerda, formando, pela simples impressão visual, um conjunto de posicionamento diferenciado das curvas referentes aos grupos fumantes. Em termos de significancia estatística de diferenças das médias obtidas entre os grupos expostos e controle, procedeu-se a um teste bicaudal de diferenças de médias (teoria das pequeras amostras) ${ }^{13}$ a niveis de confiança de $09 \%$ c $95 \%$.

T A B E L A 1

Grupos pinpulacionais de adultos da cidade de São Paulo, classificados segundo a ocupação e o hábuto de fumar.

\begin{tabular}{l|r|r|r}
\hline Grupo Populacional & Fumsintes & Não-Fumantes & Total \\
\hline $\begin{array}{l}\text { Empregados de transporte } \\
\text { Guardas de trânsito }\end{array}$ & 46 & 52 & 98 \\
\hline Total & 100 & 199 & 1 \\
\hline
\end{tabular}

T A B E L A 2

Grupo populacional de adultos da cidade de Embu-Guaçu. classificados segundo o hábito de fumar. (Grupo-Controle)

\begin{tabular}{c|c|c|c|c|c|c|}
\hline Grupo Populacional & Fumantes & Não-Fumantes & \\
\hline Policiais e empregados & 17 & 18 \\
\hline
\end{tabular}

As Figs. 1, 2, 3. 4 e 5 foram extraidas do Projeto 47/76: Avaltação do Grau de Exposição de Amostras Populacionais ao Monóxido de Carbono. Junho/1977 - STAR/DTSA-CETESB - Superintendência de Tecnologia do Ar/Diretoria de Tecnologia de Saneamento do Ar e de Controle de Fontes de Poluição - Companhid de Tecnologia de Saneamento Ambiental. 
FERnfCOLA, N. A. G. G. de \& LIMA, E. R. A valiação do grau de exposição de amostras populacionais de São Paulo (Brasil) ao monóxido de carbono. Rev. Saúde públ., S. Paulo, 13: $151-8, \quad 1979$.

T A B E L A 3

Carboxiemoglobina: Valor médio (percentagem de saturação da hemoglobina) e desvio padrâo nos grupos populacionais de adultos "expostos" da cidade de São Paulo, conforme a ocupação e o hábito de fumar.

\begin{tabular}{l|c|c}
\hline Grupo Populacional & Fumantes & Não-Fumantes \\
\hline Empregados de transporte & $4,6 \pm 1.94$ & $1,6 \pm 0,46$ \\
Guardas de trânsito & $6,3 \pm 2,07$ & $2,1 \pm 0,68$ \\
\hline
\end{tabular}

T A E E L A 4

Carboxiemoglobina: Valor médio (percentagem de saturação da hemoglobinal e desvio padrão no grupo populacional de adultos da cidade de Embu-Guaçu. conforme o hábito de fumar (Grupo-Controle)

\begin{tabular}{c|c|c}
\hline Grupo Ponulacional & Fumantes & Não-Fumantes \\
\hline Policiais e empregados & $3.8 \pm 1.74$ & $0,8 \pm 0,21$ \\
\hline
\end{tabular}

A aplicação dos testes de hipóteses acima descritos mostrou que a hipótese nula, referente à igualdade entre populações, só foi aceita no cruzamento motoristas de ônibus e grupo-controle para categoria fumante. Os demais cruzamentos tanto para fumantes como para não fumantes apresentaram rejeição da hipótese de nulidade em ambos os níveis de significância utilizados.

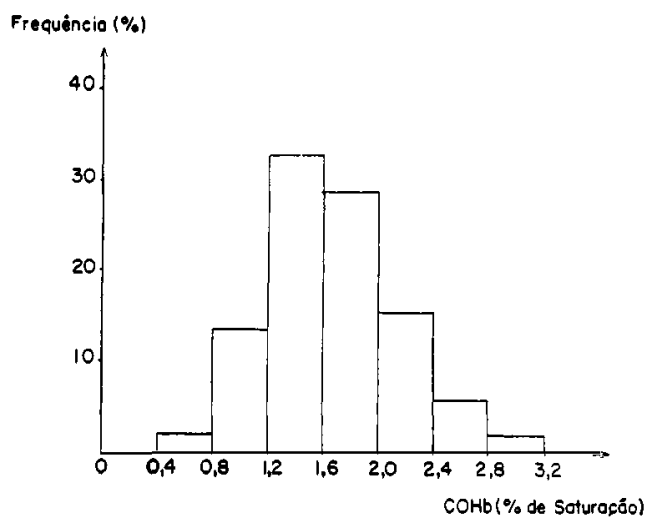

Fig. 1 - Distribuiçăo da frequència percentual dos valores de COHb (\% de saturaça) em empregados de transporte coletivo, não fumantes.

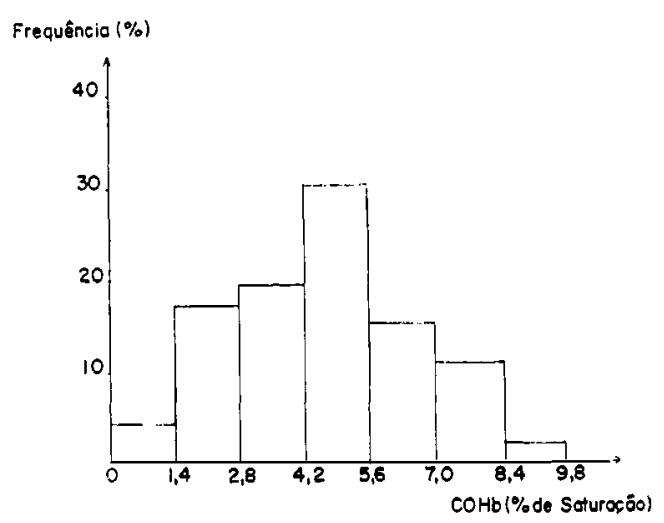

Fiğ. 2 - Distribuição de freqüência percentual dos valores de COHb (\% de saturação) em emlpregados de transporte coletivo, fumantes.

\section{DISCUSSÃO E CONCLUSÕES}

Os padrões de qualidade do ar do Estado de São Paulo para o CO são (Decreto n' 8468 de 08 de setembro de 1976): $10 \mathrm{mg} / \mathrm{m}^{*}$ (9 ppm) como média máxima de 8 horas continuas a não ser excedida mais que uma 
FERNfCOLA, N. A. G. G. de \& LIMA, E. R. A valiação do grau de expısição de amostras populacionais de São Paulo (Brasil) ao monóxido de carbono. Rev. Sá́de públ., S. Paulo, 13: $151-8,1979$.

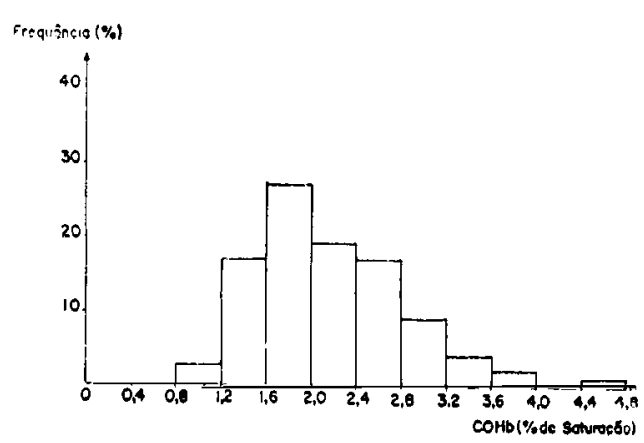

Fig. 3 - Distribuição da frequèencia percentual dos valores de $\mathrm{COHb}(\%$ de saturação) em policiais não fumantes

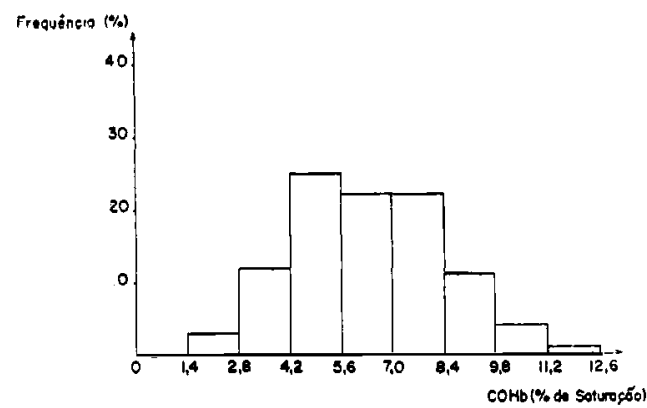

Fig. 4 - Distribuição da frequência percentual dos valores de COHb (\% de saturação) em policiais fumantes.

vez por ano; $40 \mathrm{mg} / \mathrm{m}^{3}$ (35 ppm) como média máxima de uma hora a não ser excedida mais que uma vez por ano.

Estes valores são idênticos aos adotados pela Environmental Protection Agency ${ }^{\circ}$ (EPA) dos E.U.A., propostos a 30 de janeiro de 1971. A fixação de tais padrões visou evitar a ocorrência de niveis de $\mathrm{COHb}$ superiores a $2 \%$.

No presente estudo foram propositadamente tomadas para formar o "grupo exposto" pessoas que, por força de suas atividades, servem como um provável máximo de exposição ao $\mathrm{CO}$ à qual poderia estar sujeita a população de São Paulo de forma genérica.

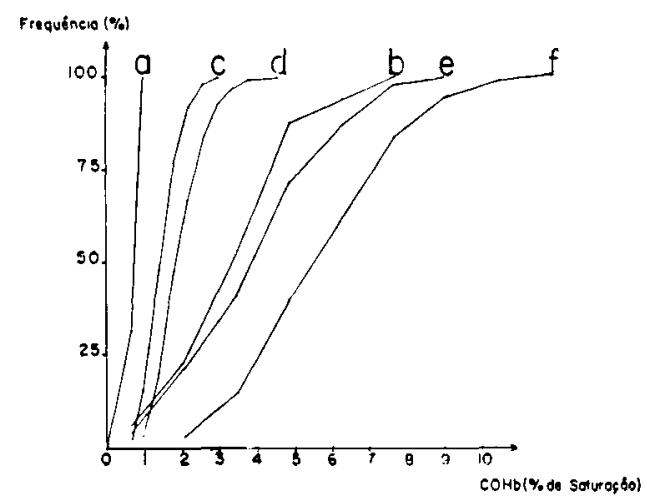

Fig. 5 - Frequência percentual acumulada dos valores de $\mathrm{COHb}$ (\% de saturação) em fumantes e não fumantes.

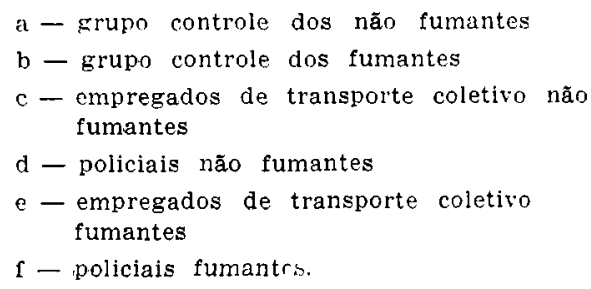

Para os grupos ftimantes, o valor máximo da mediana enco.trada foi de $6,24 \%$ de COHb para policiais de São Paulo. En estudos realizados ${ }^{15}$ foram encontradas medianas de $5,9 \%$ de $\mathrm{COHb}$ (fumantes moderados que inalam fumaça) e $6,9 \%$ de $\mathrm{COHb}$ (fumantes inveterados que fumam mais de 40 cigarros por dia e inalam a fumaça). Todavia, cumpre ressaltar que $6,24 \%$ de COHb é um valor alto, pois nem todos os policiais fumantes são inveterados e as amostras foram colhidas em torno do meio dia, quando seria pouco provável já se ter fumado 40 cigarros. Outro fator que nos prende a atenção sobre o resultado é que somente os testes de hipótese que confrontaram este grupo com os nãofumantes apresentaram rejeição da hipótese nula, isto é, mostrando que os policiais de trânsito constituem um grupo diferente dos outros testados. Cumpre ressaltar que na ocasiāo da tomada das amostras alguns elementos tinham terminado o último cigar- 
FERNiCOLA, N. A. G. G. de \& LIMA, E. R. A valiação do grau de exposição de amostras populacionais de São Paulo (Brasil) ao monóxido de carbono. Rer. Saúdo públ., S. Paulo. 13 $151-8,1979$.

ro há poucos instantes, o que fica ben evidenciado pelo desvio padrão apresentado (Tabela 3). Segundo De Bruin ${ }^{5}$, policiais fumantes expostos durante 4,5 horas nas ruas de Amsterdan apresentaram um valor médio de $5,16 \%$ de COHb, com desvio padrão de 0,27 .

Estudos referentes a niveis de $\mathrm{COHb}$ realizados en 1973 por vários pesquisadores 1.2 evidenciaram que em pacientes portadores de insuficiência coronária a inalação de $C O(50 \mathrm{ppm})$ com conseqüente elevação dos niveis de $\mathrm{COHb}$ a valores superiores a $2 \%(2,9 \%+$ e $2,68 \% 2)$ encurta significantemente o tempo até o aparecimento de dor durante a realização de exercícios fisicos.

Segundo Schlipköter 1:2 os niveis permitidos de $C O$ na atmosfera devem proteger us enfermus, pessoas idosas e crianças.

Considerando que é possivel a existência de doentes cardiacos que fiquem tão expostos quanto os policiais de trânsito nãofumantes e ụue a média de $\mathrm{COHb}$ e respectivo desvio padrão por nós ubtidos para este grupo admitem a ocorrência de valores superiores ao encontrado por Aronow c lsbell"- em seu trabalho experimental, pode-se esperar que, eventualmente, tais cardiacos apresentem problemas quando da execução de esforços físicos.

Como já mencionado, o padrão de qualidade do ar para o monóxido de carbono foi estabelecido para não se ultrapassar $2 \%$ de $\mathrm{COHb}$ em não-fumantes ". A duração da exposição e uma determinada concentração de $\mathrm{CO}$ necessária para atingir níveis de equilibrio da $\mathrm{COHb}$ é demorada. Para fins práticos, um prazo de 7 a 8 horas de exposição a uma determinada concentração estado de equilíbrio ${ }^{\top}$. Embora o presente estudo tenha observado, via de regra, o prazo acima, certamente houve variações da concentração de $\mathrm{CO}$ em cada local no periodo que antecedeu à tomada da amostra e, portanto, é impossivel qualquer correlação com estados de equilíbrio da percentagem de $\mathrm{COHb}$, bem como as concentrações locais de CO.
Em termos de comparação da percentagem de $\mathrm{COHb}$, associada ao padrão, para individuos não-fumantes, podemos dizer que: o valor médio determinado para a população de Embú-Guaçu, $0,8 \pm 0,21$, é bastante próximo aos achados por outros autores, para zonas rurais de uutros países: $0,71 \pm 0,02 i . \quad$ A diferença existente talvez possa ser explicada pelo fato de que aquele município não possui características exclusivamente rurais no tocante aos locais de coleta de amostras e ao tipo de atividade dos individuos amostradus. o valor médio de $1,6 \pm 0,46$ determinado para o grupo de empregados do transporte coletivo está dentro dos limites da percentagem de $\mathrm{COHb}$ associada ao padrão de qualidade do ar para o CO. O valor médio de $2,1 \pm 0,68$ encontrado para os guardas de trânsito supera o valor referência para a COHb.

Do ponto de vista de referencial em rela(cão ao escoamento do tráfego, admitindo que o mesmo fosse continuo sem fontes ou sumidouros, os guardas de trânsito poderiam ser tomados como referencial fixo (euleriano) enquanto os motoristas, por acompanharem a corrente, se constituiriam em um referencial móvel (lagrangeano).

No primeiro aspecto, o guarda de trânsito segue todas as variações locais de concentração. As amostras para esta população foram colhidas em diferentes partes da cidade, onde se espera, pela propria presença de policiais de trânsito, que o escoamento (o) mesmo seja problemático e, portanto, apresentando uma predominância do uso de marcha lenta, com maior emissão de monóxido de carbono pelos veículos. Os motoristas, por executarem percursos, não estariam tão expostos como os guardas de trânsito, a menos que existissem problemas de escapamento de gases para a carroceria de seu próprio veículo, o que não é de esperar. Portanto, os resultados obtidos de $\mathrm{COHb}$ para essas populações é bastante coerente ainda se levarmos em conta que 
FERAícolA. N A. G. G de \& LIMA, E. R. Avaliação do grau de exposição de amostras populacionars de São Faulo (Brasil) ao monóxido de carbono. Rer, Saúde públ, S. Paulo, 13 $151-8,1979$.

a atividade fisica de ulm policial de trânsito talvez venha a ser superior à de un empregado de transporte coletivo.

Se o padrão de yualidade do ar não deve ser ultrapassado mais que uma vez por ano, por analogia o mesmo se deveria esperar da percentagem de COHb. Ora, ambas as populaçōes do municipio de São Paulo escothidas neste estudo, se levarmos en conta os desvios padries, apresentam valores superiores a $2 \%$ de carboxiemoglobina, o que já representa um desrespeito ao valor referência. Muito embora espere-se que o restante da população deva apresentar valores menores yue $2 \%$ de carhoxiemoglobina, devemos lembrar yute tal estudi foi realizado no periudo de $10 / 11 / 76$ a $09 / 03 / 77$, relativo principalmente ao verão e cue caso fosse executado no inverno, onde em 1976 a relação das médias máximas de 8 horas (inverno/verão) foi de $16,7 / 5,2$ poderia apresentar um aumento em relaçāo ans niveis de $\mathrm{COHb}$ acui apresentados.

Em sintese, existe a necessidacte da adoção de medidas específicas em relação aos veiculos automotores e sua circulaçãu para garantir a proteção da população da cidade de São Paulo contra niveis superiores a $2 \%$ de COHb, ainda mais por haver indicios de que o referido percentual de $\mathrm{COHb}$ associado au pactrão $(9 \mathrm{ppm})$ possa ser o menor (lo) yue a aceito atualmente."

FErvicola, N. A. G. G. de \& LiMA, E. R. IEvaluation of the degree of exposure of sample population groups in $S$. Paulo (Brazil) to carbon monoxidel. Rev. Saúde públ., S. Pailo, 13:151-8, 1979.

ABstract: In order to evaluate the degree of exposure of the $S$. Panlo city (Brazil) population to carbon monoxide, 327 blood samples from adults were collected and the carboxihemoglobin content determined by the spectrophotometric method. Thirt! of these samples (control group) were taken from residents of a city (Embu-Guaçu) considered by its characteristics to be an area of low environmental carbon monoxide concentration. The following results were obtained: traffic policemen: smokers $6.3 \pm 2.07$, non-smokers $2.1 \pm 0.68$; bus drivers: smokers $4.6=1.94$, non-smokers $1.6 \pm 0.46$ : control: smokers $3.8 \div 1.74$, non-smokers $0.8 \pm 0.21$.

UNITERHS: Carbon monoxide. Spectrophotometry.

\section{REFFPENCIAS BIBLIOGRAFICAS}

1. ANDERSON. E. W. et al. Effect of Iowlevel carbon monoxide exposure on onset and duration of angina pectors. Ann. int. Hed. $79: 46-50.1973$.

2. AFIONOW. W. S. \& ISBELL, M. W. Carbon monoxlde effect on exercise-induced angma pectoris. Ann. int. Med. $\mathbf{9}$. :192-5, $197: ?$
3. BUCHWALD, $H$. A rapid and sensitive method for estimating carbon monoxide in blood and its application in problem areas. Amer. industr, Hyy. Ass. J., 30:564-9. 1969.

4. COMMINS, B. T. \& LAWTHER, P. J. A sensitive method for the determmation of carboxyhemoglobin in a finger mirk 
FERNífCOLA, N. A. G. G. de \& LIMA, E. R. A valiação do grau de exposição de amostras populacionais de São Paulo (Brasil) ao monóxido de carbono, Rev. Saúde públ., S. Paulo, 13: $151-8,1979$.

sampler of blood. Brit. J. industr. Ned., 22:139-43, 1965.

5. DE BRUIN, A. Carboxyhemoglobin jevels due to traffic exhaust. Arch. enrironm. Hlth, 15:384-9. 1967.

6. ENVIRONMENTAL PROTECTION AGENCY. National primary and secondary ambient air quality standards. Fed. Regis., Washington, D. C., 36(84) 8186,1971 .

7. KALM, A. et al, A study of carbon monoxide sources in the $S t$. Louis me'ropolitan population and some police implications. Edwardsville, Center for Urban and Environmental Rescarch and Services Southern Illinois University, 1975. (Report n" 4).

§. LAFONTAINE. A. Current views regarding the existing air quality criteria for CO. In: European Colloquium on Health Effect of Carbon Monoxile Environmental Pollution. Luxembours. 1973. Proceedings. Luxenbourg, 197:. p. 354-67.

9. MAGE, D. T. CO emission standards. J. Air Follut. control Ass., 27:10-1. 1977.
10. Miranda, J. M. et al. Carbon monoxide control in a high highway tunnel. Arch. entironm. Flth, 15:16-25, 1967.

11. ORGANIZACIÓN MUNDIAL DE LA SA. LUD. Comite de Expertos sobre la Contaminación del aire en el médio urbano, Ginebra, 1969. Informe. Ginebra, 1969. (Ser, Inf, tecn., 410).

12. SCHLIPKÖTER, H. W. Discussion. In: European Colloquium on Health Effects of Carbon Monoxide Entironmental Pollution, Luxembourg. 1973. Proceedings. Luxembourg, 1973. p. $321-2$.

1:. SPIEGEL, M. R. Estatistica. São Paulo. Ed. Me Graw-Hill do Brasil. 1972.

14. STEWART, R. D. The effect of carbon monoxide on humans. J. occup. Med., $18: 304-9.1976$.

15. U. S. Department of Health, Education and Welfare. Air quality criteria for carbon monoxide. Washington. D. C., 1970.

Recebido para publicasão em 15,01/1979 Aprovado para publicação em 22:03/1979 